\title{
Optimizing Tool Path Sequence Of Plasma Cutting Machine Using TSP Approach
}

\author{
Shreeyash Sonawane ${ }^{l, *}$, Pallavi Patil ${ }^{2}$, Ramkrishna Bharsakade ${ }^{3}$, Pankaj Gaigole ${ }^{4}$ \\ ${ }^{1}$ M.Tech (Industrial Engineering), Vishwakarma Institute of Technology, Pune, India \\ ${ }^{2}$ Director, UMAS Pvt. Ltd. Pune, India \\ ${ }^{3} \& 4$ Assistant Professor, Department of Industrial \& Production Engineering, Vishwakarma Institute of Technology, Pune, India
}

\begin{abstract}
The paper consists of optimizing the total distance traveled by the Plasma Cutting Tool Head. It is cross-functional implementation traveling salesman problem to the core manufacturing process of plasma cutting. This study contributes to reduce the total rapid traverse length (Total Through-Air Travelled Distance) by the cutting tool (Plasma Beam), which is totally a non-productive as well as non-value adding process contributing to the machine running costs and cycle time of the product directly and indirectly. For deriving this optimal sequence for the plasma cutting head, a heuristic algorithm is used. This algorithm calculates an optimal solution for a problem creating the best possible sequence. The greedy algorithm follows the problem-solving heuristics of making a locally optimal choice at every stage, finally integrating a globally optimal solution. Hence after deducing this optimal sequence and when accompanied by the Plasma Cutting tool, it resulted in the least distance traveled, contributing to minimizing cost and energy savings of the machine and simultaneously reducing the total traveled distance and similarly the cycle time.
\end{abstract}

\section{Introduction}

In the process of Plasma cutting, various critical aspects contribute significantly to the overall cycle time of the parts to be cut and also the efficiency of the machine. Out of these critical factors, the two most important factors or stages significantly impact the cutting of the metal sheets, which are Material Utilization of the sheet i.e., minimizing the scrap percentage per sheet and the Cutting Sequence ie the total tool travel required to cut the parts. The scope of the paper is focused on the second point, which is the Optimization of Cutting Sequence. The nesting is complex and dynamic process. As compared to nesting, the tool travel sequence is a factor which is ignored frequently and considered not so significant in terms of a specific time and cost savings, if calculated per sheet. This study sheds light on this niche problem and tries to deduce the optimum sequence, which, when followed, gives the least minimum tool travel distance while cutting of the part in Plasma cutting machine. The study is done through this paper deals explicitly with the Plasma Cutting machine as it has a relatively less speed of travel as compared to any Laser Cutting machine. The Plasma cutting machine uses two types of flames. 1. A Plasma Flame which consists of a mixture of Argon, Hydrogen, and Nitrogen gas, which is used to cut Stainless-Steel sheets of thickness up to 50 $\mathrm{mm}$. 2. An Oxy-fuel flame consists of a mixture of LPG and Oxygen gas, which is used in the cutting of Mild Steel sheets up to a sheet thickness on $150 \mathrm{~mm}$.
A typical plasma cutting process can take a few minutes to around a couple of hours of cutting depending upon the sheet thickness and number of parts to be cut. The time required to machine the parts depends on two factors as follows. The total distance traveled by the plasma consists of two distances- the total cutting distance (sum of the perimeter of all the parts which are to be cut) and the Rapid Traverse Length (through air travel distance while traveling from one cut part to the other). Out of these two, a lot of research has been done on reducing the machine time during actual cutting by optimizing the cutting parameters of the machine. However, a comparatively less attention was given to this second type of aspect which consists of air time travel where the cutting tool travels in an off condition completely, while moving from one cut part to the other un-cut part. This movement, if perceived from a Lean point of view, can be considered as a total non-value adding activity. The direct impact, if considered on a single nesting sheet, cannot show significant savings in terms of distance and time savings, but the cascading effect contributes to a considerable amount of savings. This distance cannot be completely eradicated though it can be reduced. It is a type of Value-Enabled activity, adding indirectly to the final finished component. The paper focuses on optimizing this distance and creating an optimum sequence model for cutting parts with the help of simple algorithms and data-handling tools.

The methodology of the paper is shown in the below flow-chart.

\footnotetext{
* Corresponding author: shreeyash.sonawane18@vit.edu
} 


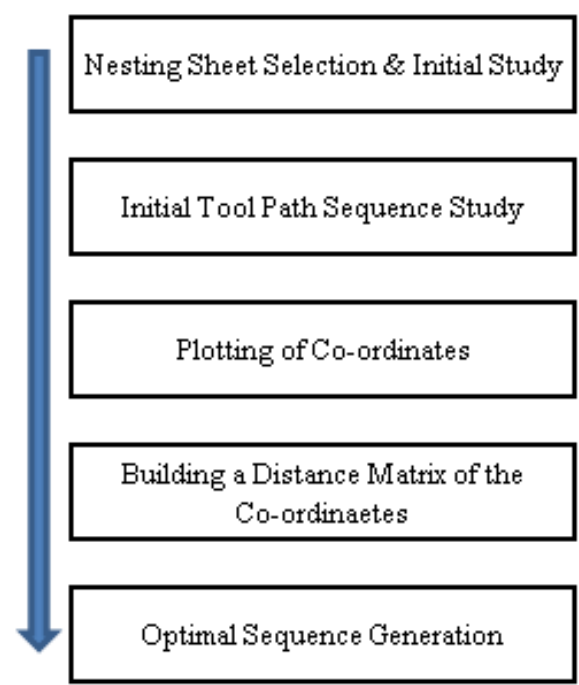

Fig. 1. Methodology Flow-Chart.

The heuristic algorithm was used to create this optimal solution by using some tools such as Excel Solver. Heuristic means "to find" or "to discover". The heuristic procedure is used to find an optimized solution and designed for solving problems quickly.

\section{Literature Review}

F. Kolahan and M. Liang [1] used a Tabu-search approach for minimizing the total processing cost for hole-making operations. The tool travel time was considered along with the tool switching time, and the cutting time and the Tabu-search algorithm was used to find the optimum solution. They attempted to reduce non-productive time using drilling or hole-making processes because they involve only holes, which can be treated as points and are much easier to showl. The problem is similar to TSP with a NP complee algorithm. This approach has been used to minimize drilling costs for a plastic injection mold. Based on the computational result, decisions regarding tool switch, tool traavel, tool selection, and machining speed specification can be simultaneously made. The result showed more than 47 percent reduction. Wen-Chen Leea, Heng Mab, and BorWen Chenga [2] studied the layout of nesting which affects the costs directly. This paper deals with the method of how efficiently a product pattern could be nested on a raw material sheet so as to maximize the utilization. The paper draws attention, to a quick location and movement (QLM) algorithm, which is applied in solving the situation of irregular shapes nested on different sheets. This approach shows two major parts: 1 . The irregular shapes are nested in a polygon. 2 . It allocates shapes by a certain logic. This deduced the results which prove that the QLM algorithm is the most easy and quickest method of all the methods. Israni and Sanders [3] in their paper considered the positioning of regular shape stocks on the sheet so that resulting in minimizing the losses and to ensure maximum production efficiency. The first fit decreasing heuristic algorithm is used, and its performance is examined. Various different algorithms are in use in the industry. Out of which many are found to be proprietary. This cutting stock problem is also used in the industries. The original form has been modified and catered according to the applications in the paper. Wang and Xie [4] in their paper studied the process-planning problem of a combined punch-laser machine blanking, based on an ant colony optimization (ACO) algorithm for optimal tool path to laser blanking processes. The result concluded that this can greately increase the operation efficiency for this type of combined punch-laser machine and appplications. Rodriques and Ferreira [5] proposed a memetic algorithm to solve the rural postman problem and proposed that it could be used for path optimization of component cutting operations. They proposed that the Rural Postman Problem (RPP) is a ttype of Arc Routing Problem (ARP). This rpp deduces a algorithm for finding out the minimum cost of a circuit on a graph. The paper shows an approach based on Memetic Algorithms - the MARP algorithm - that strives to solve the RPP and, also deals with Industrial Application, which focuses on the path optimization for cutting of components. Reginald, Pieter et al. [6] used a way of partitioning contours, which aimed at minimizing the sum of the total costs of a rooted directed minimum spanning tree to connect the partitions and the costs of traveling salesman problem (TSP) solutions within each partition. The Edmond-Liu's algorithm is used to solve the arborescence problem which is an improved version of Lin-Kernighan heuristic to solve the GTSP and simultaneously solving heuristic-repartitioning approach. With the help of which tool paths can be generated that are $4.2 \%$ faster than other which are generated by an existing tool path construction heuristic method. S Umar Sherif, N Jawahar, and Balamurali [7] Thepaper deals with generating an optimal sequence for cutting using a simutaled annealing algorithm (SAA). The proposed SAA is shown with the utilization of the optimum material. The test is carried by five different typical problems which show that this gives the best optimal sequence. The performance when compared with teo literature problems shows that the proposed SAA gives improved results than ACO and GA algorithms. Roshan D'Souza et al. [8] in their paper show the generation of optimal sequence for milling by reducing airtime through optimally connecting toolpath segments. The problem is analysed as a travelling salesman problem and solved by using a heuristic algorithm. The following algorithm has been applied in an automated process planning system, which can also be applied in other areas of path planning and optimization of laser cutting. Bennell and Oliveria [9] in their paper provided detailed explanations about a popular technique for handling geometry of nesting. The paper provides a analysis which covers the core geometric methodologies currently employed by researchers in cutting and packing of irregular shapes. It will also shed light on the theory and implementation of the approaches used for the problem of nesting of irregular parts. It provides a description of the outlook of all the researchers in the area to select the most suitable methodology. Z. M. Bi 
and Lihui [10] in their paper, shed light on the optimization of machining processes, from a different perspective of energy consumption. The paper deals with the unique relationship of energy conservation and machining attributes. The method is based on the dynamic as well kinematic behaviors of chosen machine tools. This model which is developed applied to optimize the machine setup for energy savings. [11] Jeong, C.S. and M.H. Kim, have described an approach of parallel simulated annealing for the Traveling Salesman Problem on SIMD machines. The paper describes the problem of solving the TSP on SIMD machines with linear interconnections among processing elements. Kamlapurkar and Date [12], in their paper have viewed wastage in a integrated approach and have attempted to minimize the total wastage arising from layout as well as rejections. The highly strained regions in a sheet metal blank were identified and defect map generated considering the permissible variations. The blanks are laid out, and the possible number of rejections are predicted using probability which lead to the prediction of the actual material utilization. [13] Dalalah and Khrais et al., in their paper, have described the problem of waste in irregular stock cutting of metal sheets. This problem is faced in many manufacturing companies. The approach of solving this problem was developed, which depended upon the concept of difference between the areas of a collection of polygons and the area of their convex hull. The polygon assignment inside the stock was subjected to feasibility tests so as to avoid overlapping. Costa and Gomes et al. [14] in their paper have studied three heuristic approaches are discussed. 1. The first one includes solving the case of nesting problems occurred. The experimental results which are deduced are further compared to the published results already present in the literature review along with additional results obtained from new proofs are also provided. The variants are based on the Double Lattice approach are seen to have achieved better results than the others based on the Single-Lattice approach. This is given non-convex shape and fits well with it is rotated 180. Elamvazuthi [15] solved the problem in the leather industry, for placing a set of irregularly shaped pieces on a plane irregularly shaped surface. It discussed the manual as well as automated processes of nesting and cutting for leather furniture production. This paper reported productivity improvement through automation of the leather cutting process. Tiwari and Chakraborti [16] used the GA based multi-objective optimization procedure for optimizing the layout of rectangular parts by minimizing the required sheet length (or the trim loss) as well as the number of cuts (or the total length of the cut required) to achieve the cutting process. This algorithm is used to study both guillotine and non-guillotine cutting cases. The near-optimal solution set is obtained by the evolution of Pareto-front. Jung and Ahluwalia [17] in their paper described a feature-based non-cutting tool path selection using integer programming (IP). The IP approach provides an optimum tool dispatching sequence for non-cutting path in accordance with the manufacturing rules while avoiding multi-dispatch of tools. Croes, G. A. [18] in his paper has discussed a method for solving traveling salesman problem, which he describes as finding out an efficient loop which connects a number of points in a plane. He deduced a method that has the following properties - 1. Its application to both symmetrical and asymmetrical problems with random elements. 2. Without using subjective decisions so that they can be completely mechanized. 3. It is considerably faster than any other method proposed. 4. It can be terminated at any point at which the solution obtained is accurate. Helsgaun K. [19], in his paper has described Lin Kernighan heuristic and its application, which is a successful method used for the generation of an optimal and/or near-optimal solutions for the symmetric TSP. It was found that optimal solutions have been found for the solved problems which were able to obtain a satisfied result, which included a problem which contained 13509 cities in total. Han $\mathrm{G}$ and $\mathrm{Na} \mathrm{S}$. [20], in their paper, have described two parts. 1 . The heat flowing in contour laser beam was calculated by using the finite-difference model, and a modified analytically and similarly a model was developed based on the numerical experiments. 2 . This problem consisted of a optimal torch path planning for the 20 laser cutting of a stock plate nested with Irregular parts a nesting induced a relative position of parts which was a constraint. This optimized algorithm generated a feasible cutting path sequence. This simulated annealing technique was adopted for solving the torch path optimization problem to minimize a specified cost function. Gambardella and Dorigo [21] in their paper have solved the problem of sequential ordering that extended a local search for the TSP to handle multiple constraints at a time directly without increasing the computational complexity. The algorithmwhich described a SOP-3 and Ant Colony Optimization together was described. The paper also proves that this algorithm results in a much more effective solution than the present algorithms.

\section{Problem Description}

The problem statement is a very niche kind. The aim of the study is the reduction of the non-productive process, which is the rapid traverse length, i.e., the through air travel. Conventionally the input given to the cutting sequence was based on the skill of the operator.

The plasma machine being a kind of very slow machine had a high potential in savings occurred by reduction of this total rapid traverse distance. The problem is identified as a Travellign salesman problem as the the cutting tool head compared to the travelling salesman and the parts which are to be cut as the fixed location of the customers which are needed to be visited by the salesman only once. The total distance traveled by the cutting tool head resulting in cost and energy as well as cycle time savings. 


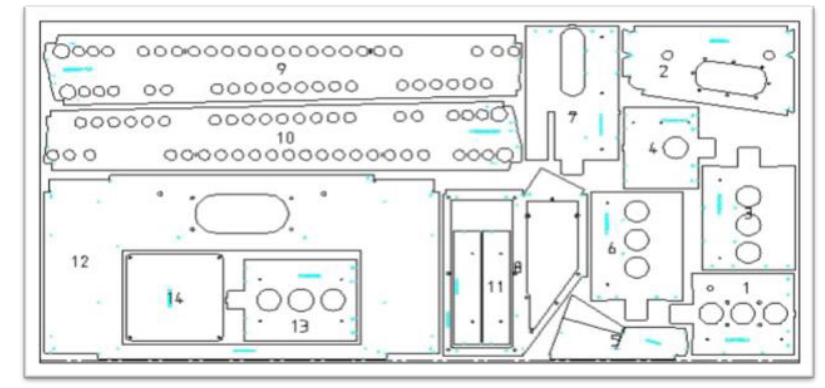

Fig. 2. Typical Nesting Sheet.

\section{Methodology}

The methodology is divided into a series of steps so as to understand the complete process in a systematic manner. The following methodology was applied to every nesting associated with the particular product, which pertained to the constraints of the process for deducing the optimum tool travel sequence. The different stages are described as follows-

\subsection{Nesting Sheet Selection and Initial Study -}

The first step for initiating the process was to study the current nesting properly. To understand the process constraints and part geometry a thorough study was made. The process constraints consist of many practical constraints and barriers which must be satisfied and adhered to while giving a nesting cut sequence. These constraints play a significant role when the sheet thickness is large, approximately exceeding $50 \mathrm{~mm}$. Hence, a particular type of nesting sheet was selected while starting the study, which fulfills the requirements of the process and does not add any obstacles in generating the sequence. The significant constraints consist of heat dissipation, which was the main reason when the operator was forced to follow a particular path so as to cut the parts without hampering the quality of the finished products.

\subsection{Initial Tool Path Sequence Study -}

To measure the change in initial and final tool travel distance, the sequence is given according to the operator's wish, and nesting is generated from the Messer software. After the nesting is generated, a pdf is created by the software automatically, which contains all the nesting information and the total tool travel distance along with the Rapid Traverse length (which is the 'airtime'). These distances are noted for further reference and calculations.

\subsection{Plotting of Coordinates -}

The first step to initiate with the generation of the optimal sequence is to know the exact coordinates of each part. These coordinates were deduced from the nesting software part by part. These coordinates were plotted on an excel sheet. To start with, a nesting with a minimum number of parts were chosen for the pilot study. The following table shows the $\mathrm{X}$ and $\mathrm{Y}$ coordinates of the start points of the cut of each part.

Table 1. Coordinates of Parts.

\begin{tabular}{|c|c|c|}
\hline Part No. & $\mathbf{X}$ & $\mathbf{Y}$ \\
\hline 1 & 1.46 & -167.15 \\
\hline 2 & 922.14 & 179.66 \\
\hline 3 & 0.24 & -548.21 \\
\hline 4 & 880.2 & -561.91 \\
\hline 5 & 1.39 & -822.92 \\
\hline 6 & 956.4 & -833.92 \\
\hline 7 & 3.79 & -930.61 \\
\hline 8 & 903.28 & -930.77 \\
\hline 9 & 965.96 & -1.61 \\
\hline 10 & 947.46 & -815.12 \\
\hline 11 & 1057.17 & -0.68 \\
\hline 12 & 1039.76 & -861.69 \\
\hline
\end{tabular}

\subsection{Building of Distance Matrix of the Coordinates -}

$$
d=\sqrt{\left(x_{2}-x_{1}\right)^{2}+\left(y_{2}-y_{1}\right)^{2}}
$$

Fig. 3. Distance Formula.

The coordinates which were captured in the previous stage were used to create a distance matrix. A distance matrix is a typical matrix of numbers (distances) representing relative distances of each and every part of each of the other parts. These distances decide at every stage where the entire sequence moves. The matrix shown below is the relative distance matrix calculated by using Excel formulas.

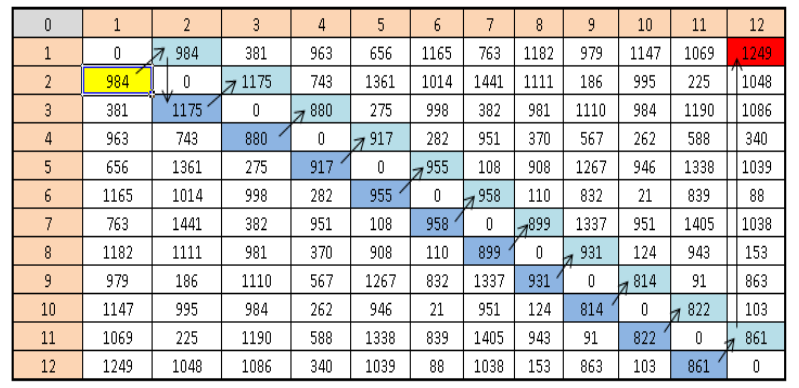

Fig. 4. Initial Relative Distance Matrix.

The arrows shown in the matrix represents the current sequence followed by the Plasma Tool Head to complete the cutting of all parts present in the nesting. The yellow box indicates the starting point, and the red box indicates the end. It is considered that the cutting is started from the first part; hence the relative distance is seen to be zero in the first box. The initial sequence followed is shown in the below table. A total of $11444 \mathrm{~mm}$ distance is traveled by the Plasma tool, as shown in the initial sequence. This distance can be further optimized in order to reduce associated costs and energy. 


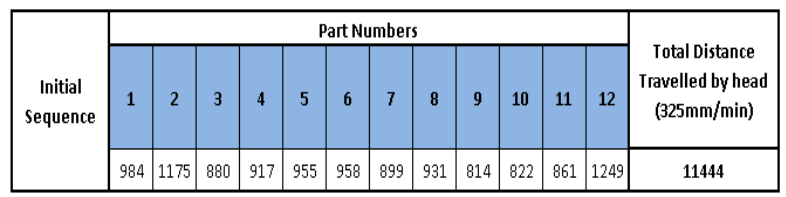

Fig. 5. Initial Sequence Followed by Cutting Tool.

\subsection{Optimal Sequence generation -}

This stage of the process of deducing the optimal sequence is of prime importance. With the help of the initial distance matrix and relative distances, by applying some constraints to the distances with the aid of an excel solver, the optimum sequence was generated. As similar to the constraints of the Travelling Salesman Problem, the same constraints were given for the cutting tool while traveling. The following are the constraints given.

- The path should be chosen in such a way that the final distance traveled should be the least.

- Each point should be visited once and only once.

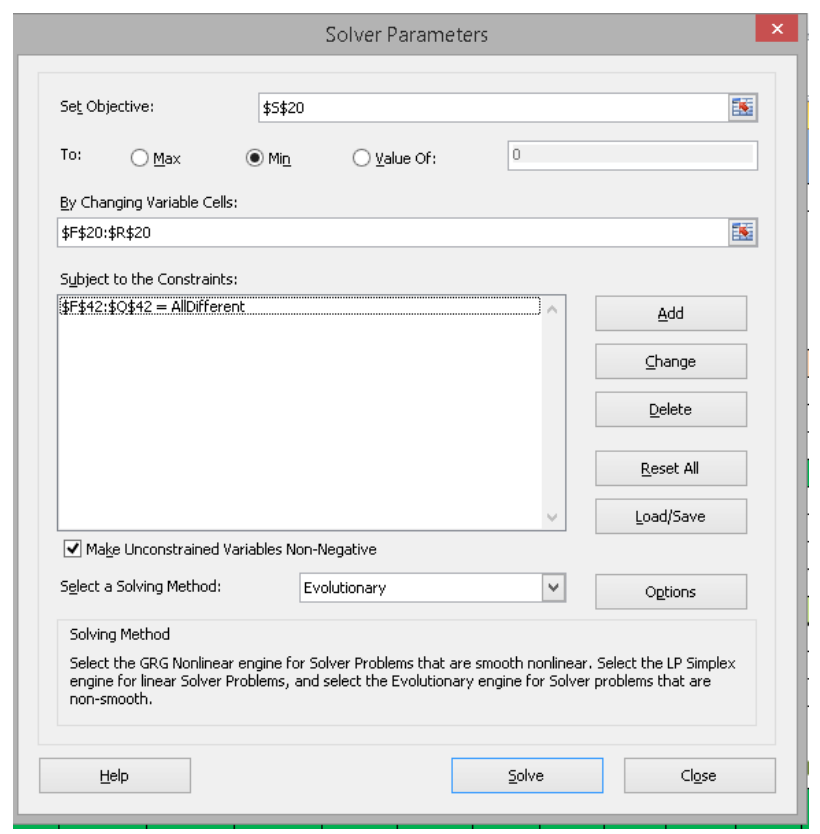

Fig. 6. Solver Parameters.

These constraints were formulated according to the solver, and corresponding functions were set in the solver. The following scree-shot from the solver will show the necessary conditions provided to it. The main objective was to reduce the total distance. Also, by changing the relative distances between the parts was the second condition given. And the third condition was that all the values should be different, which means each part to be visited only once. By subjecting the matrix to the following terms, a proper algorithm was created to guide the solver and deduce the optimum sequence for the user undergoing through many iterations. The solver took around two to three minutes to solve and iterate the optimum sequence. This time depends on the processor speed of the system used and the number of coordinates given to the solver. Below is the image of the optimum sequence generated by the solver.

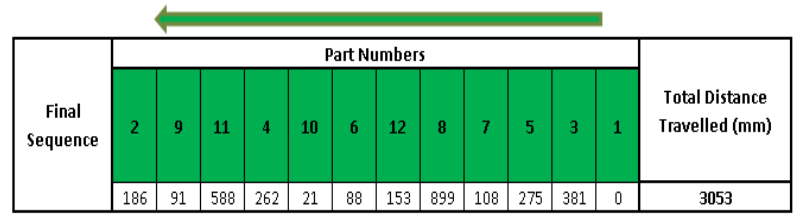

Fig. 7. Final Optimum Sequence generated.

The sequence generated is shown below in the distance matrix form to elaborate more and get more clarity on the process. Similarly, as above, the yellow box indicates the initiation of the cutting sequence, and the red box indicates the end of the cutting sequence. This sequence generated was given in the nesting software, and then the nesting sheet was generated, which showed the optimum tool path travel sequence.

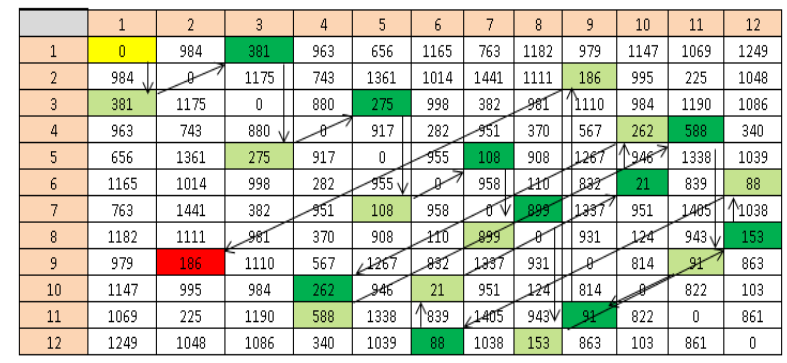

Fig. 8. Final Relative Distance Matrix.

\section{Results}

The following study shows that the previously randomly generated sequence was not optimized and still had the potential to get optimized. The initial sequence had a total tool path of $11444 \mathrm{~mm}$, whereas the optimized sequence summed up to a distance of $3053 \mathrm{~mm}$. Though specifically in the case of Plasma machines, the cutting time is high as compared to the laser machines as the plate thickness is very high. Also, at every starting point of cut, a considerable amount of time is needed to pierce the metal sheet so as to start the cutting called the piercing time. This piercing time adds up to the total time consumed for the cutting of the total parts, and this time is not always fixed. Similarly, by following the same methodology, a different nesting (nesting no 1) was selected, and the tool travel was optimized. A total of $5550 \mathrm{~mm}$ distance was saved, contributing to about 46.26 seconds. Similarly, for another nesting (nesting no 2) the total rapid traverse length was reduced from $60866 \mathrm{~mm}$ to $47449 \mathrm{~mm}$ accountable to a savings of $13417 \mathrm{~mm}$. The savings in terms of time was not high, but the corresponding savings in the distance was considerable. These minimalistic savings can show high cascading effects if captured on a long term basis. For mass production of the cutting processes, even minimum savings carry a very high value. The respective savings matrix for a different nesting is shown below. 


\begin{tabular}{|c|c|c|c|}
\hline $\begin{array}{l}\text { Final Total Rapid } \\
\text { Traverse Length } \\
\text { (mm) }\end{array}$ & 24113.2 & 2.41 & mins \\
\hline $\begin{array}{l}\text { Initial Total Rapid } \\
\text { Traverse Length } \\
\text { (mm) }\end{array}$ & 29663.90 & 2.97 & mins \\
\hline $\begin{array}{c}\text { Rapid Traverse } \\
\text { Saved } \\
\end{array}$ & 5550.7 & 0.56 & mins \\
\hline 1.50 & mins saved & \multirow{2}{*}{\multicolumn{2}{|c|}{$10000 \mathrm{~mm} / \mathrm{min}$}} \\
\hline 177.98 & (sec) Before & & \\
\hline 90.00 & (sec) After & & \\
\hline 87.98 & secs saved & & \\
\hline
\end{tabular}

Fig. 9. Savings Matrix (Nesting No 1).

\begin{tabular}{|c|c|c|c|}
\hline $\begin{array}{l}\text { Final Total Rapid } \\
\text { Traverse Length } \\
\text { (mm) }\end{array}$ & 47449.0 & 4.74 & mins \\
\hline $\begin{array}{l}\text { Initial Total Rapid } \\
\text { Traverse Length } \\
\text { (mm) }\end{array}$ & 60866.00 & 6.09 & mins \\
\hline $\begin{array}{c}\text { Rapid Traverse } \\
\text { Saved } \\
\end{array}$ & 13417.0 & 1.34 & mins \\
\hline 1.34 & mins saved & \multirow{2}{*}{\multicolumn{2}{|c|}{$10000 \mathrm{~mm} / \mathrm{min}$}} \\
\hline 365.20 & (sec) Before & & \\
\hline 284.69 & (sec) After & & \\
\hline 80.50 & secs saved & & \\
\hline
\end{tabular}

Fig. 10. Savings Matrix (Nesting No 2).

\section{Conclusion}

This paper tries to bridge the two different concepts of the supply chain and manufacturing process of cutting. The cross-functional application of the idea has a high potential for savings in manufacturing industries if worked upon correctly. The use of the Heuristic algorithm (Greedy Algorithm) proved an essential concept in providing a fast and optimum solution for deducing the Optimum Tool Travel Sequence for cutting in the Plasma machine. This sequence generated had the least possible total distance traveled by the tool resulting in distance and energy savings. The life of the motors in the machine was also increased, resulting in the total working efficiency of the machine. A savings of around 3-meter distance in the Rapid Traverse Length was derived, contributing up to an 18 percent savings in nesting no 1. A savings of approximately 13.4 meters distance in the Rapid Traverse Length was calculated, contributing to 22.04 percent savings in nesting no. 2 . The corresponding savings in time were not so significant, but if considered, the cascading effect contributes to high savings. The study carried out in the paper aimed at focusing on this very niche topic of 'Airtime' travel of the Plasma Cutting Tool Head and successfully tried to justify and optimize the distance and the associated savings.

\section{References}

1. F. Kolahan, M. Liang. International Conference on Computers \& Industrial Engineering. Vol. 31. 371 374. (1996)

2. W. C. Lee, H. Mab, B. W. Cheng. Computer-Aided Design. Vol 40. 625-633. (2008)

3. S. Israni, J. Sander. Journal of Manufacturing Systems. Vol I. 169-82. (1982).

4. G. G. Wang, S. Q. Xie. International Journal of Production Research. Vol. 43, No. 11. 21952216.(2005)

5. A. M. Rodrigues, J. S. Ferreira. International Journal of Combinatorial Optimization Problems and Informatics. Vol. 3. 22-37. (2012)

6. R. Dewil. P. Vansteenwegen, D. Cattryssea, M. Lagunab, and T. Vossen. International Journal of Production Research. Vol. 53, No. 6. 1761-1776. (2015)

7. U. Sherif, N. Jawahar, and M. Balamurali. Journal of Manufacturing Systems. Vol 33. 624-638. (2014)

8. K. Castelino, R. D'Souza, and P Wright. Journal of Manufacturing Systems. Vol. 22 No. 3. 173-180. (2002)

9. J. A. Bennell, J. F. Oliveria, European Journal of Operations Research. Vol 184. 397-415. (2008)

10. Z. M. Bi, L. Wang. Journal of Manufacturing System. Vol 31. 420-428. (2012)

11. C. S. Jeong, M. H. Kim. Parallel Computing. Vol 17. 221-228. (1991)

12. R. L. Kamalapurkar, P. P. Date. Journal of Material Process and Technology. Vol 177. 81-3. (2006)

13. D. Dalalah, S. Khrais, and K. Bataineh. Journal of Manufacturing Systems. Vol 33. 27-40. (2014)

14. M. T. Costa, A. M. Gomes, and J. F. Oliveira. European Journal of Operations Research. Vol 192. 29-40. (2009)

15. I. Elamvazuthi, S. Kamarudding, and M. S. Azmi. International Journal Mechanical \& Mechatronics Engineering. Vol 9. 25-29. (2009)

16. S. Tiwari, N. Chakraborty. Journal of Materials Processing Technology. Vol 173. 384-393. (2006)

17. J. Y. Jung, R. S. Ahluwalia. Journal of Manufacturing Systems. Vol 13. 165-76. (1994)

18. G. A. Croes. Operations Research 6 (6). 791-812. (1958)

19. K. Helsgaun, European Journal of Operational Research. Vol 126 (1) 106-130. (2000)

20. G. C. Han, S. J. Na. Journal of Manufacturing Processes 1 (1). 62-70. (1996)

21. L. M. Gambardella, M. Dorigo. INFORMS Journal on Computing. Vol. 12, No. 3. 237-255. (2000) 